\title{
Adoecimento e religiosidade/espiritualidade uma possível reflexão
}

\author{
Addiction and religiosity/spirituality a possible reflection
}

\section{Fernanda Sampaio Novaes', Claudio Luiz Anunciação Santos Júnior², Paula Silva Peixoto³, Gabriel Leonardo de Jesus Lima Santos ${ }^{4}$, Thaís Marques Moura ${ }^{5}$, Gilmara Ribeiro Santos Rodrigues ${ }^{6}$}

${ }^{1}$ Escola Bahiana de Medicina e Saúde Pública. Salvador, Bahia, Brasil. ORCID: 0000-0002-4552-1198. fernandanovaes16.1@bahiana.edu.br ${ }^{2}$ Escola Bahiana de Medicina e Saúde Pública. Salvador, Bahia, Brasil. ORCID: 0000-0002-2640-9045. claudiojunior16.1@bahiana.edu.br

${ }^{3}$ Escola Bahiana de Medicina e Saúde Pública. Salvador, Bahia, Brasil. ORCID: 0000-0001-6938-571X. paulapeixoto16.1@bahiana.edu.br ${ }^{4}$ Escola Bahiana de Medicina e Saúde Pública. Salvador, Bahia, Brasil. ORCID: 0000-0003-2714-4349. gabrieljsantos2015.2@bahiana.edu.br ${ }^{5}$ Escola Bahiana de Medicina e Saúde Pública. Salvador, Bahia, Brasil. ORCID: 0000-0001-9667-4875. thaismoura16.1@bahiana.edu.br ${ }^{6}$ Autora para correspondência. Escola Bahiana de Medicina e Saúde Pública. Salvador, Bahia, Brasil. ORCID: 0000-0001-9667-4875. gilmararodrigues@bahiana.edu.br

RESUMO | OBJETIVO: Refletir sobre o papel da religiosidade/ espiritualidade no processo de adoecimento. MÉTODO: Trata-se de um ensaio teórico-reflexivo apresentado a partir da importância da religiosidade/espiritualidade relacionada ao processo de adoecimento e às práticas de cuidado em saúde. Realizado nos meses de setembro e outubro de 2019, a partir de estudos e discussões de um grupo de discentes do curso de enfermagem que atuam em um projeto de extensão que presta assistência a pessoas com hipertensão arterial e diabetes mellitus em um ambulatório de saúde de uma instituição privada de ensino superior. RESULTADOS: A religiosidade/espiritualidade tem auxiliado na aceitação de prognósticos negativos decorrentes da cronicidade de doenças, fazendo com que a pessoa doente ressignifique o processo do adoecimento. CONCLUSÃo: Deste modo, a religiosidade/espiritualidade pode ser utilizada como recurso que auxilia na adesão ao tratamento, cuidados de saúde em geral e como auxiliar no prognóstico de saúde.

PALAVRAS-CHAVE: Espiritualidade. Cooperação e adesão ao tratamento. Assistência integral à saúde.
ABSTRACT | OBJECTIVES: To reflect on the role of religiosity /spirituality in the disease process. METHOD: This is a theoretical-reflective essay presented from the importance of religiosity/ spirituality related to the process of illness and health care practices. Held in September and October 2019, based on studies and discussions of a group of nursing students working in an extension project that provides assistance to people with hypertension and diabetes mellitus in a health clinic of a private higher education institution. RESULTS: Religiosity/ spirituality has helped in the acceptance of negative prognoses resulting from the chronicity of diseases, making the sick person reframing the disease process. CONCLUSION: Thus, religiosity/ spirituality can be used as a resource that assists in adherence to treatment, health care in general and as an aid in health prognosis.

KEYWORDS: Spirituality. Cooperation and adherence to treatment. Comprehensive health care. 


\section{Introdução}

Esta reflexão apresenta como ponto de partida a religiosidade/espiritualidade como objeto de pesquisa das ciências da área da saúde e visa refletir sobre o papel da mesma no processo de adoecimento. Assim, a partir da questão: Como a religiosidade/espiritualidade tem sido abordada no processo de adoecimento e no cuidado com a saúde? resolveu-se realizar este trabalho que tem como objetivo refletir sobre o papel da religiosidade/espiritualidade no processo de adoecimento e no cuidado com a saúde.

O envelhecimento da população é um fato observado em todo o mundo, tendo com consequência, o grande número de indivíduos com doença crônica. Partindo do pressuposto que estes agravos e as suas consequências não são precisos, estáticos e nem breves, e que perpassam o cotidiano de quem os vivencia, há necessidade de direcionar o nosso olhar para a compreensão de todos os fatores que participam deste processo de adoecimento e das formas de abordagens dos profissionais de saúde, incluindo o aspecto religiosidade/espiritualidade.

Trata-se de um ensaio teórico-reflexivo oriundo de estudos e debates pertinentes a partir da perspectiva da religiosidade/espiritualidade como objeto de auxilio e estruturação do cuidado em saúde. Realizada nos meses de setembro e outubro de 2019, por um grupo de discentes do curso de enfermagem que atuam em um projeto de extensão que presta assistência a pessoas com hipertensão arterial e diabetes mellitus em um ambulatório de saúde uma instituição privada de ensino superior da cidade de Salvador-Bahia-Brasil.

No Brasil ainda predomina a medicina cartesiana, modelo de tratamento de saúde fragmentado por especialidades médicas e que na maioria das vezes deixa de proporcionar ao indivíduo um atendimento integral e sistematizado em uma única consulta. Assim, existe uma aceitação maior e uma melhor adesão ao tratamento quando diferentemente do modelo de assistência hegemônico, é ofertado um cuidado de saúde sem focar necessariamente na cura da doença e que contempla à pessoa em seus muitos aspectos biopsicossociais e espirituais'.
$\mathrm{Na}$ atualidade surgem diversas práticas assistenciais que visam o indivíduo na sua totalidade e integralidade. O focar no cuidado em saúde considerando o indivíduo em sua totalidade, incluindo o que se refere à espiritualidade, faz com que o modelo tradicional hegemônico seja cada vez mais questionado.

A saúde é considerada um bem-estar de vida, composto por múltiplos aspectos que compreendem o indivíduo: aspectos emocionais, físicos, sociais, psíquicos e espirituais, que devem ser contemplados sem distinção de raça, de religião, ideologia política ou condição socioeconômica². Desta maneira, o cuidado em saúde não pode ser prestado sem olhar o indivíduo de forma integral e os múltiplos aspectos que compõem o referido bem-estar devem estar em equilíbrio para que a saúde seja restabelecida1.

Neste contexto, as ações em saúde têm perdido sua dimensão cuidadora e, apesar de encontrarmos a abordagem de temas como religião, religiosidade e espiritualidade no âmbito científico, elas têm perdido potência e eficácia ${ }^{3}$. Podemos perceber que a ciência sozinha tem se mostrado restrita para resolver todos os problemas de saúde do indivíduo e, consequentemente podemos observar pouca adesão à maioria dos tratamentos, sobretudo de pessoas com adoecimento crônico.

Ao se considerar a vivência da dimensão religiosa/ espiritual como multidimensional, apenas conhecer a religião dos usuários atendidos não é o suficiente, torna-se necessário entender o processo de construção de suporte fornecido pela espiritualidade/religiosidade e suas implicações na vida do indivíduo ${ }^{4}$. A dimensão espiritual ou religiosa para a saúde vai além de uma confissão religiosa, não dependendo de lugar, tempo ou códigos que a definam ${ }^{5}$. O indivíduo em tratamento não pode ser separado de suas dimensões cognitiva, afetiva, sociocultural, biológica, sob o risco de prejudicar o cuidado integral ${ }^{3}$.

Ampliar o debate sobre as implicações da religiosidade e espiritualidade no contexto da saúde é imprescindível, uma vez que não restam mais dúvidas sobre a associação entre esses elementos ${ }^{5}$. Para tanto, é importante definirmos o conceito de espiritualidade que consideramos nesta reflexão e que também permeia o conceito de saúde. 
A religiosidade e a espiritualidade possuem significados distintos, mas geralmente são erroneamente definidos como sinônimos. Neste ensaio, por não termos como objetivo discutir o sentido dos dois termos, as duas terminologias serão abordadas como um conceito integrado com o intuito de promover uma melhor compreensão da temática.

A espiritualidade como algo que dá sentido à vida, que além de satisfação também traz coragem para encarar contratempos e vicissitudes terrenas. E que nos ajuda a respeitar o próximo, a entendê-lo, mesmo em momentos que o outro possa ser incorreto $^{6}$. A espiritualidade é uma força invisível, de grande importância nas vidas das pessoas. É uma dimensão que implica no cuidado integral aspetos físicos, emocionais e sociais, a essência do seu ser6. Sendo esta dimensão reconhecida como fonte de bem-estar e de qualidade de vida. Espiritualidade é a busca inerente de cada pessoa pelo significado e propósito definitivo da vida e influencia as percepções sobre a noção de saúde. O que pode ou não estar relacionada a propostas de uma determinada religião ${ }^{7}$.

A religiosidade/espiritualidade está ancorada na cultura dos brasileiros e faz parte do senso comum, influenciando as crenças, comportamentos e visão de mundo. Assim, consiste no caminho que a pessoa encontra para transcender o adoecer, as perdas, a internação e o vazio existencial. Consiste em uma importante estratégia de resiliência no existir do indivíduo e contribui para o enfrentamento de patologias, da solidão, entre outras demandas significativas, que colaboram para a diminuição do bem-estar ${ }^{9}$.

A abordagem da dimensão da espiritualidade da pessoa adoecida exige preparo por parte da família e dos profissionais de saúde e deve englobar o cuidado integral, cujo determinante da qualidade de vida abrange o bem-estar mental e espiritual, para além dos aspetos físicos, emocionais e sociais, é uma necessidade e essência do $\operatorname{Ser}^{10}$.

Visualizar a religiosidade/espiritualidade como uma necessidade humana básica pode servir como um indicador positivo para a saúde, como por exemplo, a Fé, que resulta em esperança, e assim pode ajudar na adesão ao tratamento, permitindo reconhecer que a mesma pode repercutir na melhoria da saúde do indivíduo ${ }^{8}$. A religiosidade/espiritualidade tem auxiliado na aceitação de prognósticos negativos decorrentes da cronicidade de doenças, fazendo com que a pessoa doente ressignifique o processo do adoecimento. A religiosidade/espiritualidade, como mecanismo de enfrentamento, é capaz de aliviar a dor, o estresse psicológico e, muitas vezes, a sensação de fracasso ${ }^{11}$. Através da espiritualidade pode-se enfrentar $\mathrm{o}$ adoecimento sem perder a esperança ${ }^{12}$.

Estudos demonstram que as pessoas que têm crenças religiosas e que as associam nas suas condições de saúde e doenças apresentam melhores condições de aceitar as alterações no seu processo de adoecimento. Assim, a religiosidade/espiritualidade pode ser considerada como um fator de proteção para os fatores de risco à saúde e consequentemente como auxiliar ao tratamento ${ }^{3}$.

Todavia, deve-se levar em consideração que a vivência da religiosidade/espiritualidade em alguns casos pode não favorecer ao tratamento, mas ao contrário, dificultar à adesão do indivíduo ao mesmo *. Os profissionais de saúde, especialmente a psicóloga e a enfermeira, devem ajudar a pessoa a ver se sua religiosidade está favorecendo ou não à sua recuperação.

Nota-se a dificuldade de a equipe de saúde abordar temáticas que sejam consideradas tabus para a sociedade, como por exemplo a sexualidade e a religiosidade/espiritualidade. Isso podemos observar inclusive nas organizações de saúde que têm o processo de enfermagem sistematizado, quando na maioria das vezes, os itens que aproximam esses dois temas não são abordados.

Vale ressaltar, que apesar do reconhecimento crescente dos benefícios da assistência espiritual e dos profissionais de saúde, em sua maioria psicólogas e enfermeiras, reconhecerem o cuidado espiritual como primordial no processo de adoecimento, percebemos que essa abordagem é ineficiente e raramente é fornecida da forma como deveria. Essa dificuldade quase sempre é justificada pela falta de conhecimentos, competências e preparo, pela falta de tempo, pelo medo de invadir a intimidade da pessoa, assim como pelo receio de não ser capaz 
de lidar com as questões levantadas, divergências religiosas e pela falta de consciência acerca da própria espiritualidade ${ }^{10}$.

Notamos que a experiência religiosa/espiritual do indivíduo aos cuidados em saúde carece de sistematização prática fundamentada e reconhecida organizacionalmente, principalmente ao valorizarmos a saúde baseada em evidências. É importante acolhermos a religiosidade/espiritualidade das pessoas que estão sob o nosso cuidado e de seus respectivos familiares, dando-Ihes um melhor suporte e integrando essa dimensão no cuidado em saúde ${ }^{3}$.

Assim, cabe salientar a contribuição e a importância para a formação em enfermagem fundamentada no pensamento holístico e sistêmico, onde o holístico busca a visão da totalidade de modo integral e o sistêmico, procura relacionar o conhecimento das partes com o conhecimento do todo e vice-versa ${ }^{13}$.

A limitação deste ensaio consiste no tipo de estudo, do qual as reflexões descritas não podem ser tomadas como verdades contundentes e generalizáveis, visto também, que apesar do grande número de publicações que afirmam o benefício da religiosidade/ espiritualidade na manutenção ou recuperação da saúde do indivíduo, existem correntes cientificamente opostas que questionam acerca de qualquer benefício ou mudança no estado de saúde.

\section{Conclusão}

Teceram-se reflexões acerca da importância de contemplarmos o indivíduo em sua totalidade e integralidade e da abordagem da religiosidade/espiritualidade no contexto hospitalar, tão quanto a necessidade de instrumentalizar os profissionais de saúde para lidar com a dimensão religiosa/espiritual do usuário, com o intuito de prestar um cuidado de qualidade.

A religiosidade/espiritualidade é considerada uma dimensão do cuidar e tem uma forte relação com vários aspetos da saúde, o que ressalta a relevância em valorizá-la no prognóstico da doença.
Apesar da limitação expressa, a discussão da temática traz contribuições para a área da enfermagem, saúde e política pública, considera-se que as reflexões apresentadas são relevantes para a valorização da Saúde baseada em evidências e pela incipiente abordagem da religiosidade/espiritualidade entre os profissionais de saúde brasileiros.

\section{Agradecimentos}

A Profa Dra Cátia Suely Palmeira e a graduanda de enfermagem Gabriella da Cruz Silva Dias.

\section{Contribuições dos autores}

Novaes FS, Santos Júnior CLA, Peixoto PS, Santos GLJL e Moura TM foram responsáveis pela concepção do estudo e pesquisa dos artigos. Rodrigues GRS foi responsável pela concepção do estudo, redação e revisão dos artigos.

\section{Conflitos de interesses}

Nenhum conflito financeiro, legal ou político envolvendo terceiros (governo, empresas e fundações privadas, etc.) foi declarado para nenhum aspecto do trabalho submetido (incluindo mas não limitando-se a subvenções e financiamentos, participação em conselho consultivo, desenho de estudo, preparação de manuscrito, análise estatística, etc.).

\section{Referências}

1. Rodrigues KM. Terapias Integrativas e Complementares: itinerário terapêutico e espiritualidade, uma possível reflexão. Revista Contraponto. 2015;2(1):1-15.

2. Organização Mundial de Saúde. Constituição da Organização Mundial da Saúde. Documentos básicos, suplemento da $45^{\mathrm{a}}$ edição. [Internet]. 2006. Disponível em: http://www.who.int/ governance/eb/who_constitution_sp.pdf

3. Silva TD'O; Marques LF. Dossiê Espiritualidade e Saúde. Problematizações, Desafios e Possibilidades da inserção da religiosidade/espiritualidade ( $r / e)$ no contexto hospitalar brasileiro. Interações - Cultura e Comunidade. 2016;11(20):98114. 
4. Henning-Geronasso MC; More CLOO. Influência da

Religiosidade/Espiritualidade no Contexto Psicoterapêutico. Psicol

Cienc Prof. 2015;35(3):711-725. doi: 10.1590/1982-3703000942014

5. Gomes NS, Farina M, Forno CD. Espiritualidade, religiosidade e religião: reflexão de conceitos em artigos psicológicos. Rev Psicol IMED. 2014;6(2):107-112.

6. Lourenço I. A espiritualidade no processo terapêutico. Coimbra: Quarteto; 2004 .p. 136.

7. Chochinov, H. M. (2004). Dignity and the eye of the beholder. J Clin Oncol. 2004;22(7):1336-40. doi: 10.1200/ JCO.2004.12.095PUCHALSKI, CM. 2006

8. Puchalski CM. A time for listening and caring: Spirituality and the care of the chronically ill and dying. Oxford: Oxford University Press; 2006. p. 480.

9. Menezes, TMO. Dimensão espiritual do cuidado na saúde e enfermagem. Editorial. Rev Baiana Enferm. 2017;31(2):e22522. doi: $10.18471 /$ rbe.v31i 2.22522

10. Malhão, MIP. A dimensão da espiritualidade do doente em fim de vida [Dissertação]. Bragança: Instituto Politécnico de Bragança, Escola Superior de Saúde; 2018.

11. Rocha ACAL. A espiritualidade no manejo da doença crônica do idoso [Dissertação]. Escola de Enfermagem da Universidade de São Paulo, São Paulo; 2011.

12. Puchalski C, Ferrell B, Virani R, Otis-Green S, Baird P, Bull J et al. Improving the quality of spiritual care as a dimension of palliative care: the report of the consensus conference. J Palliat Med. 2009;12 (10):885-904. doi: 10.1089/ jpm.2009.0142. PMid:19807235

13. Santos SSC, Hammerschmidt KSA. Complexity and the reconnection of interdisciplinary knowledge: contribution of Edgar Morin's thoughts. Rev Bras Enferm. 2012;65(4):561-5. doi: 10.1590/S0034-71672012000400002 